\title{
Oxydation du phenol sur un catalyseur à base de Fer supporté sur une argile marocaine
}

\author{
Noura LAHBABI ${ }^{\text {* }}$, Zakia RAIS ${ }^{2}$, M. HAJJAJI ${ }^{3}$ ef S. KACIM ${ }^{3}$ \\ 'Laboratoire d'ingénierie des matériaux organométalliques et moléculaire. Faculté des \\ Sciences Dhar El Mehrez, Fès, Maroc. \\ ${ }^{2}$ Laboratoire d'analyses physico-chimiques et matériaux catalytiques Faculté des \\ Sciences Dhar El Mehrez, Fès, Maroc. \\ ${ }^{3}$ Université Cadi Ayyad, faculté des sciences Semlalia, Marrakech, Maroc.
}

*Correspondance,courriel ${ }^{(2)}$ : raiszakia@yahoo.fr

\section{Résumé}

L'objectif du présent travail est l'étude de l'oxydation par voie humide du phénol en catalyse hétérogène, en présence de peroxyde d'hydrogène. Les catalyseurs choisis sont à base de fer et de cuivre; l'oxydation est effectuée dans des conditions normales de température et de pression. Les résultats expérimentaux montrent une oxydation complète du phénol et une diminution significative de la demande chimique en oxygène $D C O$. D'autre part ces catalyseurs manifestent une grande stabilité; on peut donc les utiliser sans causer des problèmes de pollution métallique.

Mots-clés : Phénol, peroxyde d'hydrogène, catalyse hétérogène, Fe-argile, oxydation.

\section{Abstract \\ Oxydation of phenol on a catalyst containing iron supported on a Moroccan clay}

Oxidation of phenol by hydrogen peroxide over Fe-clay catalysts, was investigated at normal temperature and pressure, and with $5 \mathrm{gL}^{-1}$ catalyst. The experimental results showed complete oxidation of phenol and significant $D C O$ removal under mild working conditions. Moreover, a high stability of the catalysts was fownd. Compared whith processses using homogenous catalysis, the possibility of induced pollution caused by the metal ions in the solution is avoided.

Keywords : Phenol, hydrogen peroxide, heterogenous catalysis, Fe-clay, oxidation. 


\section{Introduction}

Les effluents aqueux pollués par la matière organique provenant de l'industrie chimique et de l'activité agricole, sont très peu biodégradables du fait de la grande concentration en polluant (DCO de $500 \mathrm{mg}$ à $1500 \mathrm{mg} / \mathrm{L}$ ).

Les effets nocifs de la pollution par les phénols entraînent la dégradation de la faune et de la flore ainsi les poissons, en particulier les espèces à chaire grasse : (anguilles, saumons, truites .....) accumulent les phénols et peuvent en contenir jusqu'à $30 \mathrm{mg} / \mathrm{kg}$, ils sont alors impropres à la consommation.

Les procédés d'oxydation en phase liquide semblent apporter une solution efficace et moins coûteuse. Sur ce principe,

Alejandre et coll [1,2] ont étudié l'activité catalytique du cuivre et du nickel dans la réaction d'oxydation du phénol en milieu aqueux; ils ont montré que ces catalyseurs sont très actifs en oxydation. Le taux de conversion du phénol dépend des conditions de préparation du catalyseur et la réaction d'oxydation est très sélective en $\mathrm{CO}_{2}$.

Des catalyseurs à base de métaux lourds supportés sur des argiles (montmorillonites) [3], de la silice [4] ont montré une grande activité catalytique dans l'oxydation du phénol par le peroxyde d'hydrogène. A pression atmosphérique et $\mathrm{T}=80^{\circ} \mathrm{C}$, la dégradation du phénol est totale et rapide $(15 \mathrm{mn})$ avec une sélectivité de minéralisation de $93-59 \%$. Les auteurs ont signalé une lixiviation de métal de $6 \%$.

Matos et coll [5] ont effectué une oxydation photocatalytique du phénol à l'aide d'une suspension de $\mathrm{TiO}_{2}$ et de charbon actif; ils ont montré que l'addition du charbon actif $\mathrm{sur}^{\mathrm{TiO}_{2}}$ induit un phénomène de synergie en augmentant l'efficacité de la photocatalyse.

La cinétique de décomposition du phénol par le peroxyde d'hydrogène sous rayonnement $\mathrm{U}$ V est étudiée par M.Rodriguez et coll [6], B.Tryba et coll [7]. Sur des catalyseurs à base de fer : $\mathrm{Fe}_{-}-\mathrm{TiO}_{2}$ et $\mathrm{Fe}$-C- $-\mathrm{TiO}_{2}$, le produit d'oxydation est le chatéchol. Ce dernier joue un rôle très important dans l'accélération de la vitesse d'oxydation du phénol.

La réaction de photo-Fenton en phase hétérogène a été étudiée par $F$. Martinez et coll [8]. L'élimination du phénol en solution aqueuse est effectuée à température ambiante, sous irradiation UV-vis $(313 \mathrm{~nm})$ à un $\mathrm{pH}$ neutre. La concentration en peroxyde d'hydrogène joue un rôle important dans la stabilité du catalyseur. A $0.5 \mathrm{~g} / \mathrm{L}$ du catalyseur, un abattement total du phénol, ainsi qu'une remarquable minéralisation organique sont observés.

Barrault et coll [9] ont effectué une oxydation catalytique du phénol par le peroxyde d'hydrogène sur des argiles pontées par des espèces mixtes (Al-Cu), et ont montré que la teneur en cuivre et la méthode de préparation du catalyseur avaient une grande influence sur l'activité catalytique.

Une étude originale sur le traitement en phase hétérogène d'une solution phénolique par le peroxyde d'hydrogène avec le Fe-ZSM-5 comme catalyseur a été effectuée par Fajerwerg et 
coll [10]. A $90^{\circ} \mathrm{C}$ et sous pression atmosphérique une élimination totale du phénol et une diminution significative du COT ont été obtenues.

Falcon et coll [11] ont développé dans leur laboratoire le procédé WPO : "Wet Peroxide. Oxidation"; ils ont montré que les systèmes utilisant le $\mathrm{Fe}, \mathrm{Cu}, \mathrm{Mn}$ en phase homogène permettent une oxydation complète des acides carboxyliques dans les conditions suivantes: Tı $100^{\circ} \mathrm{C}, \mathrm{pH}=3, \mathrm{P}=\mathrm{latm}$, temps de la réaction lh, la quantité stæchiométrique de $\mathrm{H}_{2} \mathrm{O}_{2}$ est 1,5. Dans ces conditions ils ont montré qu'il y a un effet de synergie entre ces métaux.

Pour notre part nous nous sommes intéressés à ce procédé WPO d'épuration par oxydation catalytique mais en phase hétérogène; ce procédé fait appel au peroxyde d'hydrogène comme oxydant, les catalyseurs étant le $\mathrm{Fe}$ et le Cu supportés sur une argile marocaine. Dans cet article, seuls les résultats obtenus avec le fer sont représentés.

\section{Matériel et méthodes}

\section{2-1. Matériaux utilisés}

L'argile que nous avons utilisée, notée $M B$ (provenant de Moulay Brahim, région de Marrakech) a été caractérisée par A. Alami [12], il a montré que la fraction argileuse $(<2 \mu \mathrm{m})$ est constituée de minéraux à faciès fibreux (palygorskite et sépiolite ). Le minéral dominant étant la palygorsquite ( $86 \%$ ).

Les minéraux associés sont : le quartz, la dolomite, et la calcite.

Les différentes caractéristiques de l'argile sont données dans le tableau suivant :

Tableaul : Caractéristiques physico-chimiques de l'argile MB selon [12].

\begin{tabular}{|c|c|c|c|}
\hline $\begin{array}{c}\text { Surface spécifique } \\
\left(\mathrm{m}^{2} / \mathrm{g}\right)\end{array}$ & CEC $(\mathrm{meq} / \mathrm{l00 \textrm {g }})$ & Minéraux argileux & Minéraux associés \\
\hline 100 & 32 & $86 \%$ palygorsquite & $8 \%$ Calcite \\
& & sépiolite & $36.5 \%$ Dolomite \\
& & & $24.5 \%$ Quartz \\
\hline
\end{tabular}

CEC : Capacité d'échange cationique en milliéquivalent par 100 grammes d'argile.

\section{2-1-1. Préparation des catalyseurs par imprégnation.}

Nous avons choisi l'argile MB à l'état naturel, comme support catalytique à cause sa grande surface spécifique et de sa capacité d'échange cationique. 
La méthode d'imprégnation que nous avons utilisée est celle citée dans la littérature [13]. Les catalyseurs ainsi préparés sont séchés à l'étuve pendant une nuit. Le précurseur qui a été utilisé est : $\left(\mathrm{FeCl}_{3}, 6 \mathrm{H}_{2} \mathrm{O}, \mathrm{M}=270.30\right)$.

Une série de catalyseurs a été préparée, en faisant varier le taux du métal imprégné de 1 à $5 \%$ (le pourcentage est massique). Pour préparer un gramme de catalyseur, les concentrations en précurseurs métalliques sont les suivants :

Tableau2 : Concentrations en précurseurs métalliques, nécessaires pour préparer un gramme de catalyseur

\begin{tabular}{|c|c|}
\hline Catalyseur & {$\left[\mathrm{FeCl}_{3}, 6 \mathrm{H}_{2} \mathbf{0}\right](\mathrm{mol} / \mathrm{I})$} \\
\hline $1 \% \mathrm{Fe}-\mathrm{MB}$ & $3,5810^{-2}$ \\
\hline $2.5 \% \mathrm{Fe}-\mathrm{MB}$ & $8,9410^{-2}$ \\
\hline $5 \% \mathrm{Fe}-\mathrm{MB}$ & $17,910^{-2}$ \\
\hline
\end{tabular}

\section{2-1-2. Caractérisation des catalyseurs}

Les catalyseurs préparés par imprégnation du métal sur l'argile sont caractérisés par diffraction des rayons $X$ (RX) à l'aide d'un diffractomètre PHILIPS PWI710 (tube en cuivre $\left., \lambda_{\mathrm{k} \alpha}:=1,54060\right)$.

Pour les catalyseurs imprégnés par le fer, l'augmentation du taux d'imprégnation n'apporte aucune modification à la structure du support, aucune phase nouvelle n'est détectée, les diffractogrammes des catalyseurs sont superposables à celui du support, quelque soit le taux en fer imprégné. Cela nous a conduit à conclure que le fer se trouve bien dispersé sur l'argile.

\subsubsection{Lavage des catalyseurs}

Dans le but de connaître la quantité du métal qui est effectivement retenue par l'argile naturelle $M B$, nous avons effectué un lavage des catalyseurs: $5 \mathrm{~g} / \mathrm{L}$ de catalyseurs préparés par imprégnation du fer sur l'argile MB sont lavés à l'eau ultra pure pendant $24 h$ sous agitation. Après centrifugation, nous avons dosé la quantité du fer qui passe dans les eaux de lavage. 
Tableau3 : Dissolution du fer dans /'eau de lavage en $\mathrm{mg} / \mathrm{L}$ et en $\mathrm{mg} / \mathrm{g}$ de catalyseur.

\begin{tabular}{|c|c|c|c|}
\hline Catalyseurs & {$[\mathrm{Fe}]$ en $\mathrm{mg} / \mathrm{L}$} & {$[\mathrm{Fe}]$ en $\mathrm{mg} / \mathrm{g}$ de cat } & Teneur réelle en fer \\
\hline $1 \% \mathrm{Fe}-\mathrm{MB}$ & 0.25 & 0.05 & $0.99 \%$ \\
\hline $2.5 \mathrm{Fe}-\mathrm{MB}$ & 2 & 0.4 & $2.46 \%$ \\
\hline $5 \% \mathrm{Fe}-\mathrm{MB}$ & 1.9 & 0.38 & $4.96 \%$ \\
\hline
\end{tabular}

D'après les résultats du Tableau 3, l'argile MB représente un véritable piège du métal fer. La teneur réelle en métal fixée sur l'argile, est pratiquement égale à la teneur théorique. Ces résultats sont en accord avec la diffraction RX qui montre que le fer se trouve bien dispersé sur l'argile.

\section{2-2. Techniques analytiques et méthode opératoire}

\section{2-2-1. Méthode opératoire}

Pour réaliser les essais selon le procédé WPO, la réaction est effectuée en régime discontinu. Sur $100 \mathrm{ml}$ d'une solution phénolique $5.10^{-4} \mathrm{M}$, on ajoute $0,5 \mathrm{~g}$ de catalyseur lavé; l'introduction de l'agent oxydant $\left(5 \mathrm{ml} \mathrm{de} \mathrm{H}_{2} \mathrm{O}_{2} \quad 10^{-3} \mathrm{M}\right)$ correspond au début de la réaction d'oxydation. L'oxydation est effectuée dans les conditions normales de température et de pression. C.N.T.P.

Des prélèvements au cours du temps sont effectués pour avoir : l'évolution du pH, de la concentration en oxydant, en phénol, et en DCO.

\section{2-2-2. Techniques analytiques}

le dosage du peroxyde d'hydrogène dans les solutions mères et pour des concentrations supérieures à $10^{-3} \mathrm{~mol} / \mathrm{L}$ est effectué selon la méthode iodométrique. Pour des concentrations comprises entre $10^{-5} \mathrm{~mol} / \mathrm{L}$ et $10^{-3} \mathrm{~mol} / \mathrm{L}$, la méthode d'Eisenberg est utilisée [14].

Le dosage de fer est effectué par absorption atomique.

Les sous produits sont analysés par chromatographie en phase liquide haute pression HPLC; la séparation des composés est effectuée sur deux types de colonnes : pour l'analyse de composés aromatiques; nous avons utilisé une colonne $C 18$ Nucléosil de longueur $I=25 \mathrm{~cm}$, de diamètre $4.6 \mathrm{~mm}$, la phase mobile est $60 \%$ eav, $40 \%$ méthanol.

Pour la séparation des acides une avons fait appel à une colonne AminexHPX-87(Biorad), de longueur I $=30 \mathrm{~cm}$, de diamètre $7.8 \mathrm{~mm}$, la phase mobile est $\mathrm{H}_{2} \mathrm{SO}_{4}: 0.005 \mathrm{~mol} / \mathrm{L}$.

La mesure du carbone organique total (COT) est effectuée à l'aide d'un appareil DohrmannDC 190.

La demande chimique en oxygène $D C 0$ est mesurée selon la méthode décrite dans [15]. 


\section{Résultats et discussion}

3-1. Oxydation du phénol par le peroxyde d'hydrogène en présence de catalyseur : Oxydation en présence de catalyseurs Fe-Argile

Nous avons effectué préalablement un essai d'adsorption du phénol sur cette argile; les résultats montrent que le taux d'élimination du phénol par adsorption ne dépasse pas $20 \%$.

Des prélèvements ont été effectués au cours du temps, pour suivre l'évolution du pH, de la concentration en substrat, de la $D C O$, du COT, de la quantité du métal qui passe en solution.

\subsubsection{Evolution du pH au cours de l'oxydation : effet de la concentration en métal sur l'argile}

Les résultats sont ceux de la Figure 1

Pour les deux catalyseurs, le pH reste constant au début de l'oxydation ensuite il diminue légèrement, en raison de la formation d'acides organiques acide oxalique, acide formique) au cours de l'oxydation du phénol.

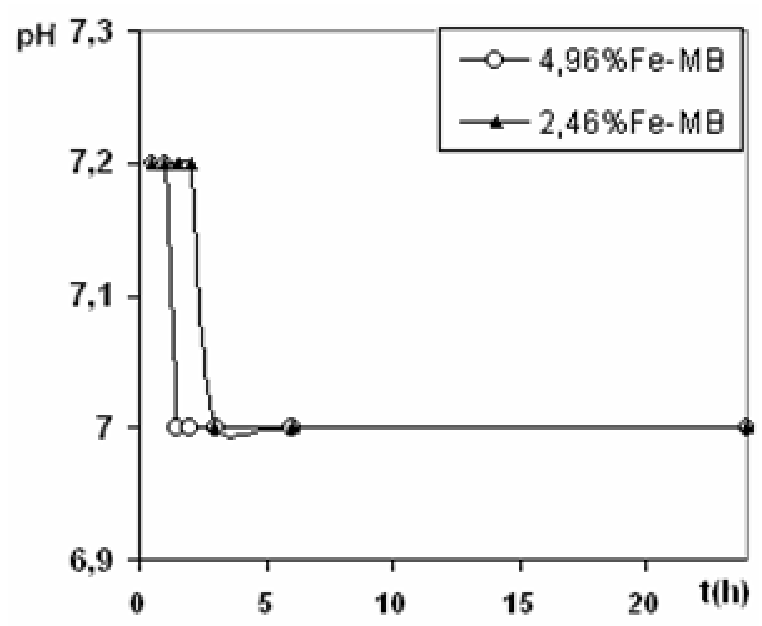

Figure 1 : Evolution du $\mathrm{pH}$ : Effet de la teneur en fer du catalyseur. $\left[\mathrm{H}_{2} \mathrm{O}_{2}\right]: 10^{3} \mathrm{M}$, [phénol]: $5.10^{4} \mathrm{M}$, [cat] : $5 \mathrm{~g} / \mathrm{ll}$, C.N.T.P. 


\section{3-1-2. Décomposition de l'agent oxydant : influence de la quantité du métal imprégnée sur l'argile}

Le système hétérogène qu'on a utilisé est très actif, il permet une activation totale de $\mathrm{H}_{2} \mathrm{O}_{2}$. Les résultats de décomposition de $\mathrm{H}_{2} \mathrm{O}_{2}$ sont dans le tableau suivant :

Tableau4 : Influence de la quantité du métal imprégnée sur I'argile sur la décomposition de $\mathrm{H}_{2} \mathrm{O}_{2}$

\begin{tabular}{|c|c|c|c|}
\hline catalyseur & \multicolumn{3}{|c|}{$\% \mathrm{de}_{2} \mathrm{O}_{2}$ éliminé } \\
\hline $4.96 \% \mathrm{Fe}-\mathrm{MB}$ & 95 & 100 & 100 \\
\hline $2.46 \% \mathrm{Fe}-\mathrm{MB}$ & 90 & 100 & 100 \\
\hline Temps & $\mathrm{lh}$ & $5 \mathrm{~h}$ & $24 \mathrm{~h}$ \\
\hline
\end{tabular}

On remarque une décomposition très importante de l'agent oxydant dès la première heure de la réaction, cette décomposition résulte vraisemblablement de l'existence de réactions parasites: réaction de terminaison qui existe entre les différents radicaux $\left(\mathrm{OH}^{\bullet}, \mathrm{HO}_{2}^{\bullet}, \mathrm{O}_{2}^{\bullet} \ldots\right)$.

\section{3-1-3 Elimination du phénol : influence de la quantité du métal imprégnée sur l'argile}

Les résultats de la Figure2 montrent qu'au début de la réaction, le pourcentage d'oxydation du phénol augmente avec la quantité du métal imprégnée sur l'argile, la vitesse de la réaction d'oxydation est donc proportionnelle au nombre de sites catalytiques. Après lh d'oxydation l'élimination du phénol s'élève jusqu'à $100 \%$ et devient indépendante du taux de métal imprégné.

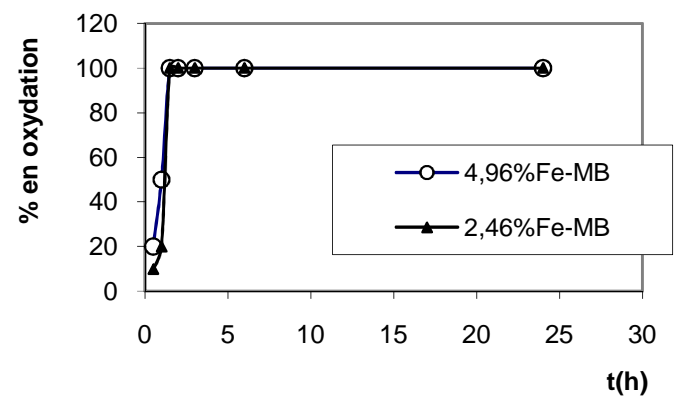

Figure 2 : Elimination du phénol: Effet de la teneur en fer du catalyseur. $\left[\mathrm{H}_{2} \mathrm{O}_{2}\right]: 10^{3} \mathrm{M}$, [phénol: ]:5.10 M, [cat]: $5 \mathrm{~g} / \mathrm{ll}$, C.N.T.P. 


\section{3-1-4. Elimination de la DCO : influence de la quantité du métal imprégnée sur l'argile.}

Nous définissons la diminution de la demande chimique en oxygène comme étant :

$D C O(\%)=\left[D C 0^{\circ}-(D C O)^{t} /(D C O)^{\circ}\right] \cdot 100$

$(D C O)^{\circ}$ : est la valeur de la DCO avant introduction du catalyseur

Les résultats obtenus sont regroupés sur la figure suivante :

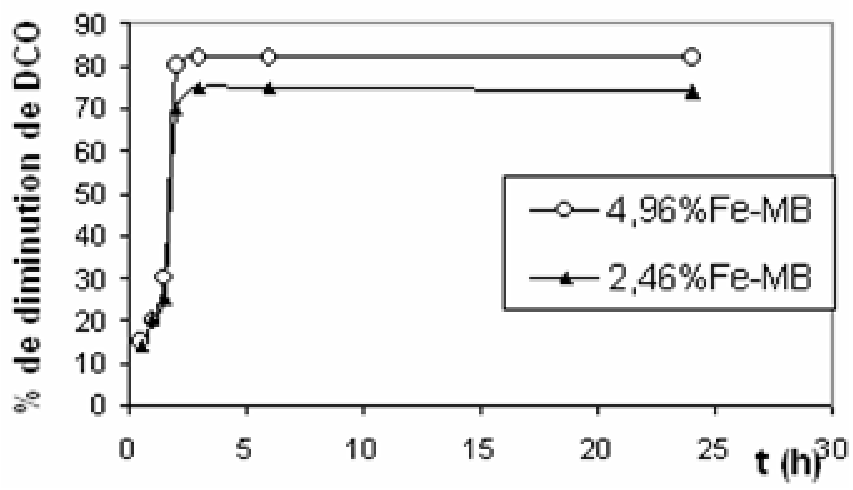

Figure 3 : Elimination de la DCO : Effet de la teneur en fer du catalyseur. $\left[\mathrm{H}_{2} \mathrm{O}_{2}\right]: 10^{3} \mathrm{M}$, [phénol]:5.10 $\mathrm{M}$, [cat] : $5 \mathrm{~g} / \mathrm{l}$, C.N.T.P.

On pensait que la différence d'activité en fonction de la teneur en métal serait très importante, or les résultats qui figurent ci-dessus permettent de conclure que le taux d'élimination de la $D C O$ dépend très légèrement de la quantité du métal imprégnée sur l'argile, les sites catalytiques seraient donc formés à partir d'une faible quantité de fer sur la surface argileuse. Un résultat analogue a été signalé par N.Al.Hayek [16], lors de l'oxydation du phénol par le peroxyde d'hydrogène en présence de catalyseur $\mathrm{Fe}$ Alumine.

Une autre remarque à signaler est que la vitesse de disparition du phénol est supérieure à celle de la $D C O$, cela est due à la formation et à l'accumulation des produits intermédiaires issus de la réaction d'oxydation au cours du temps.

\section{3-1-5. Elimination du COT}

Un prélèvement d'un échantillon après deux heures d'oxydation est effectué pour

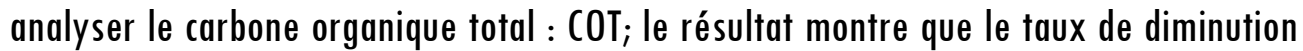
du COT est de 60\%. pour le catalyseur 4.96\% Fe-MB. La différence entre le taux d'abattement du COT et celui de la DCO, vient du fait que le phénol n'est pas oxydé totalement en $\mathrm{CO}_{2}$. Le $\mathrm{COT}$ restant dans le milieu réactionnel correspond surtout à l'acide oxalique qui est réfractaire à l'oxydation dans les conditions opératoires utilisées. 


\section{3-1-6. Stabilité des catalyseurs}

Dans le but de connaître la stabilité des catalyseurs utilisés, nous avons effectué un dosage de la quantité du fer qui passe en solution au cours de l'oxydation; le résultat montre que les catalyseurs sont d'une stabilité suffisante pour être utilisés sans causer de problèmes de pollution métallique (la concentration en fer détectée dans la solution ne dépasse pas $0,3 \mathrm{mg} / \mathrm{L}$ soit une dissolution de métal de $1 \%$ ).

\section{3-1. Discussion}

Les sous - produits d'oxydation sont analysés par chromatographie HPLC. Pour les catalyseurs à base de fer le produit final de l'oxydation est l'acide oxalique.

Dans le but de confirmer les résultats obtenus en présence de catalyseur solide en milieu aqueux et de bien montrer que l'oxydation n'était pas due au relargage du fer dans la solution, un test catalytique à blanc est effectué en phase homogène, dans les mêmes conditions que l'oxydation en présence de catalyseur. Img de fer est introduit sous forme de nitrate de fer ferrique. Les résultats montrent que le taux du phénol éliminé en oxydation homogène ne dépasse pas $15 \%$ et celui de la DCO est nul. En conclusion, les résultats obtenus en présence de catalyseur solide montrent bien que la réaction d'oxydation se passe essentiellement en phase hétérogène et cela à cause de la bonne stabilité et activité des catalyseurs.

\section{Conclusion}

Cette étude avait comme objectif la recherche de catalyseurs stables en milieu aqueux et permettant l'oxydation du phénol en phase hétérogène par le peroxyde d'hydrogène. Pour cela nous avons préparé différents catalyseurs supportés sur une argile marocaine. Notre choix s'est porté sur le précurseur de fer : élément choisi dans l'imprégnation.

Les résultats montrent que les catalyseurs à base de fer sont potentiellement actifs en oxydation du phénol. Après Ih d'oxydation, nous avons pu éliminer la totalité du phénol. Le taux d'élimination de la DCO est de $80 \%$, un abattement en COT de $60 \%$ est atteint.

Les catalyseurs utilisés ont montré une grande stabilité en milieu aqueux, ils sont donc assez favorables pour être utilisés sans causer des problèmes de pollution métallique. 
Une analyse par HPLC a montré que la réaction d'oxydation est sélective en acide oxalique.

Les résultats que nous avons exposés sont encourageants, nous pensons pouvoir orienter la réaction jusqu'à l'oxydation totale du phénol en dioxyde de carbone, et cela en étudiant la réaction à différentes valeurs de pH. D'autre part, pour une meilleure utilisation de l'agent oxydant, nous pensons faire la réaction d'oxydation en régime continu.

\section{Références}

[1] - A. Alejandre, F. Medina, A. Fortuny, P. Salagre, J.E. Sueiras, Applied catalysis B: Environmenta/16 (1998) 53-67.

[2] - A. Alejandre, F. Medina, P. Salagre, A. Fabregat J.E. Sueiras, Applied catalysis B : Environmenta/ 18 (1998) 307-315.

[3] - M.N. Timofeeva, S.Ts. Khankhasaeva, S.V. Badmaeva, A.L. Chuvilin, E.B. Burgina, Applied Catalysis B : Environmental,59, (2005) 243-248.

[4] - N. Crowther, F. Larachi; Applied Catalysis B. Environmental. 46, (2003) 293305.

[5] - J. Matos, J. Laine, J. Marie Hermann, Applied Catalysis B : Environmenta/, 18 (1998) 281-291

[6] - M. Rodriguez, N. Ben.Abderrakik, S. Contreras, E. Chamarro, J. Gimenez, et S. Splugas, Catalysis B: Environmenta/37 (2002) 131-137.

[7] - B.Tryba, W. Antoni, M. Inagakiet M. Toyoda, Applied catalysis B : Environmental, 63 (2006) 215-221

[8] - F.Martinez, G. Calleja, J. A.Melero et R. Molina, Applied catalysis B : Environmental, 60(2005) 181-190.

[9] - J. Barrault, C. Bouchoule, K. Echachoui, N. Frini-Srasra; Applied catalysis B : Environmental, 15 (1998) 269-274.

[10] - K. Fajerwerg, J.N. Foussard, A. Perrard, et H. Debellefontaine, Wat. Sci. Technol., 4 (1997) 103-110.

[11] - M. Falcon, K. Fajerwerg, J.N. Foussard, E. Puech-Costes, M.T. Maurette et H. Debellefontaine, Environmental technology 16 (1995) 501-513.

[12] - A. Alami, Caractérsation physico-chimique d'argiles marocaines. Etude de leur capacité de rétention, Diplôme d'étude supérieure DES. Faculté des sciences de Marrakech, Maroc (1997). 
[13]- N. Lahbabi, A.Kherbeche, A.Chakroune, M.Hadji, L'eau. L'industrie. Les nuisances vol I (1999) 123-129.

[14] - G.M. Eisenberg, Ind.Eng.Chem, 15 (5) (1943) 327-328.

[15] - J. Rodier; I'analyse de l'eav; 8ième édition. Dunod (1996).

[16] - N. AL Hayek, Oxydation catalytique en milieu aqueux de composés aromatiques et d'acides organiques par le peroxyde d'hydrogène. Thèse de doctorat. Université de Poitiers, (1985). 